\title{
Formación en competencias laborales generales, desafío para la educación superior
}

Training in general occupational skills, a challenge for higher education

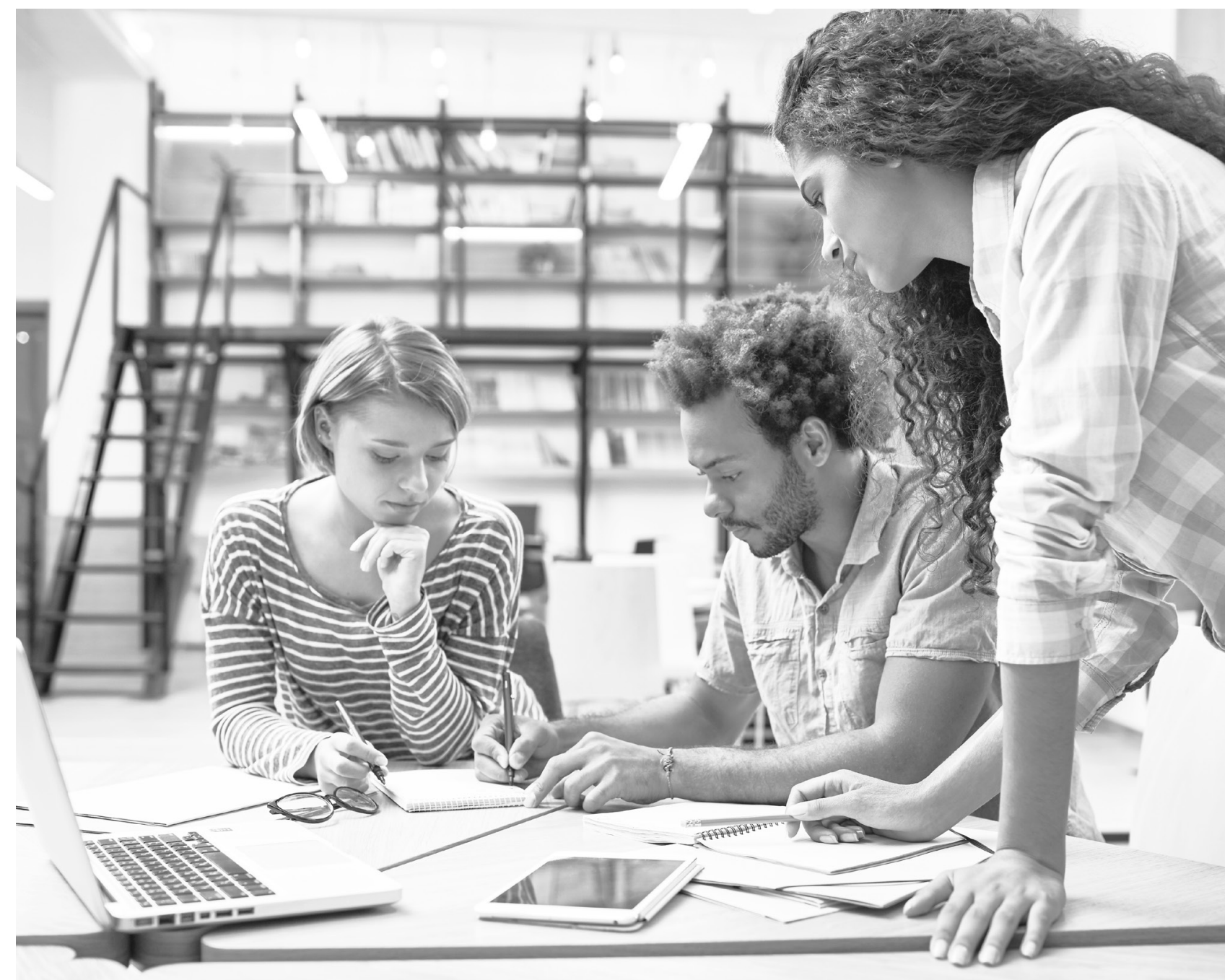




\title{
Formación en competencias laborales generales, desafío para la educación superior ${ }^{1}$ Training in general occupational skills, a challenge for higher education
}

\author{
Javier Francisco Rueda Galvis ${ }^{2}$, Socorro Astrid Portilla Castellanos ${ }^{3}$
}

Artículo recibido en septiembre 19 de 2019; artículo aceptado en noviembre 15 de 2019

\begin{abstract}
Este artículo puede compartirse bajo la Licencia Creative Commons Atribución-NoComercial-Compartirlgual 4.0 Internacional y se referencia usando el siguiente formato: Rueda, J. F. \& Portilla, S. A. (2020). Formación en competencias laborales generales, desafió de la educación superior. I+D Revista de Investigaciones, 15 (1), 40-48. DOI: https://doi.org/10.33304/revinv.v15n1-2020004
\end{abstract}

\begin{abstract}
Resumen
Para las instituciones educativas de nivel superior, su principal premisa se enfoca en la formación integral de sus estudiantes desde diversos enfoques pedagógicos. Esto implica modelos curriculares basados en el desarrollo de competencias en las que se da prioridad a la cualificación profesional y la interdisciplinariedad, y se desconoce que actualmente la demanda laboral exige fuertemente la formación en competencias blandas que permitan al futuro profesional desenvolverse de manera eficiente y eficaz en el sector laboral real. Por tal razón, el presente documento expone un estudio evaluativo bajo el esquema de revisión documental, a fin de proporcionar un análisis del entorno académico y organizacional, acerca de las características más importantes que en términos de competencias blandas demanda el mercado laboral. Lo anterior, con el propósito de mejorar las estrategias formativas y ofrecer mayores niveles de productividad por parte de los egresados a las empresas y a la sociedad en general.
\end{abstract}

Palabras clave: Competencias laborales, habilidades blandas, habilidades duras, desempleo en Colombia.

\begin{abstract}
For higher level educational institutions, the main premise is centered on the integral training of students from various pedagogical approaches. This implies curricular models based on the development of skills, of which priority is given to professional qualification and interdisciplinarity, ignoring that at present, labor demand strongly requires training in soft skills that allow the future professional to function efficiently and effectively in the actual working sector. For this reason, this document presents an evaluative study under the documentary review scheme, in order to provide an analysis of the academic and organizational environment about the most important characteristics that the labor

\footnotetext{
${ }^{1}$ Artículo de revisión, de enfoque cualitativo, resultado de un proyecto de investigación terminado, perteneciente al área de competitividad empresarial, subárea gestión del talento humano, desarrollado en el grupo de investigación PORTER, fue financiado por la Universidad de Investigación y Desarrollo (Bucaramanga, Colombia). Dirección: calle 9 n. ${ }^{\circ}$ 23-55. PBX: 6352525. Fecha de inicio: febrero de 2019. Fecha de terminación: septiembre de 2019.

${ }^{2}$ Ph.D. en Administración de Empresas, integrante del grupo de investigación PORTER, profesor-investigador de la Universidad de Investigación y Desarrollo (Bucaramanga, Colombia). Dirección: calle 9 n. ${ }^{\circ}$ 23-55 PBX: 6352525. ORCID ID: https://orcid.org/0000-0002-2795-7844. Correo electrónico institucional: jrueda31@udi.edu.co.

${ }^{3} \mathrm{Ph}$.D. en Educación, integrante del grupo de investigación FIELDS, profesora-investigadora de la Universidad de Investigación y Desarrollo (Bucaramanga, Colombia). Dirección: calle 9 n. 23-55 PBX: 6352525. ORCID ID: https://orcid.org/0000-0002-7580-5634. Correo institucional: sportilla2@udi.edu.co.
} 
market demands in terms of soft skills. All of this in an attempt to improve the training strategies in graduates and, thus, offer higher levels of productivity to companies and society in general.

Keywords: Occupational skills, soft skills, hard skills, unemployment in Colombia.

\section{Introducción}

Las competencias laborales generales están definidas por el Ministerio de Educación Nacional (2005) como "el conjunto de conocimientos, habilidades, actitudes y valores que un estudiante debe desarrollar para desempeñarse de manera apropiada en cualquier entorno productivo, sin importar sector económico, actividad, cargo, complejidad o grado de responsabilidad requerido". Este concepto se incorpora en el sistema colombiano desde el año 2006. A partir de esta propuesta, se vincularon acciones para propiciar las capacidades laborales y el espíritu de emprendimiento desde la formación media con proyección al nivel superior, que se conoce actualmente como la "Guía N. 21 para la articulación de la educación superior con el mundo productivo", que se constituye como el marco argumental para el diseño de programas curriculares (Águila, Mejía, Pérez, Rivera \& Ramírez, 2015).

Este documento presenta la diferencia entre competencias laborales específicas y generales, explicando las razones para su desarrollo, clases y caracterización, con el objetivo de ofrecer alternativas para la formación integral de estudiantes en el ámbito de habilidades duras y blandas relacionadas con el emprendimiento y la productividad laboral (Águila et al., 2015). En este sentido, la idea es contar con herramientas metodológicas con las que las instituciones diseñen currículos que respondan a las expectativas laborales reales del mercado laboral, $y / 0$ en competencias para el emprendimiento a nivel de secundaria, técnico y profesional, con estrategias pedagogías innovadoras y creativas que contribuyan al desarrollo socioeconómico sostenible del país y a incrementar la competitividad empresarial (Vera, 2016).

Por tanto, el presente documento reflexiona sobre el papel de las instituciones de formación superior en Colombia frente a las competencias que demanda el mercado laboral actual, bajo un análisis crítico de los proyectos educativos institucionales PEl y el desempeño profesional de los egresados. Es importante que se generen con este tipo de documentos espacios de reflexión y crítica para promover el fortalecimiento de la oferta educativa en términos de mejores espacios académicos, a fin de garantizar egresados con perfiles laborales más adaptados a las necesidades del sector real, mediante competencias blandas en un mundo globalizado y cambiante.

En este sentido, el presente documento se enfoca en poder contribuir a generar argumentos que justifiquen por qué las instituciones de educación superior deben incluir en los programas de formación técnica y/o profesional competencias blandas que fortalezcan las capacidades laborales generales de los egresados, para lograr una mejor inserción en el mercado laboral y fomentar el espíritu emprendedor. Se busca con estos aportes analizar la pertinencia y apropiación que necesita la formación superior en términos de nuevas competencias laborales generales, dentro del amplio espectro de programas curriculares que ofertan las universidades. Para este fin, es preciso promover nuevas líneas de investigación que contribuyan a la generación de nuevo conocimiento y un perfil de profesionales aptos para las necesidades del sector empresarial (Banco de la República, 2019).

\section{Metodología}

Para el desarrollo de este artículo, se utilizó el modelo de investigación evaluativa, bajo el esquema de revisión documental, a fin de generar análisis y reflexiones críticas soportadas en argumentos bibliográficos retrospectivos asociados con cada uno de los temas de mayor relevancia para la academia, tanto en el ámbito nacional como en el internacional. Lo anterior, mediante una exploración documental de carácter relevante que argumenta datos como opiniones validas frente a la necesidad de desarrollar mayores competencias blandas en los estudiantes a nivel técnico y profesional, a fin de generar autoevaluaciones y debates en las instituciones de educación superior, con base en las evidencias aquí descritas (Guirao, 2015).

En términos generales, este artículo presenta un estudio exploratorio de carácter crítico que se soporta en los diversos postulados de orden científico que analizan el modelo de formación actual frente a las teorías que se proponen para los centros de educación superior, tanto en Colombia como en Latinoamérica. Estas se postulan como elementos explicativos que correlacionan en forma directa el nivel de desarrollo de las competencias aprendidas frente a las altas tasas de desempleo que vive actualmente el país, aspecto que resulta ser un fenómeno que afecta en mayor medida a la población de hombres y mujeres jóvenes, con edades que oscilan entre los 18 y los 28 años (Departamento Administrativo para la Prosperidad Social, 2019).

Con base en este esquema, la investigación inició con la enunciación de postulados que condujeran a la 
concreción de una pregunta de investigación, sobre la cual se estructura el desarrollo del marco conceptual y el análisis de los datos obtenidos. Por tanto, se planteó como el eje temático la siguiente pregunta de investigación: ¿Por qué las instituciones de educación superior en Colombia deben implementar programas con énfasis en la formación de competencias laborales generales?

\section{Resultado de la revisión}

\section{Desempleo e informalidad en Colombia}

En Colombia, el tema de disminuir el nivel de desempleo sigue siendo el compromiso pendiente más importante por parte del Gobierno nacional, problemática que se desencadena principalmente por el limitado crecimiento económico, la globalización de los mercados, la migración venezolana y las poco eficaces políticas para impulsar el emprendimiento y un mayor desarrollo empresarial que vincule mano de obra calificada (Banco de la República, 2019).

Lamentablemente, este panorama resulta cada vez más preocupante, ya que durante los últimos 19 años la tasa de desempleo anual promedio en el país superó los dos dígitos porcentuales, con un valor promedio anual del $11 \%$. Esta situación se mantiene hasta el primer semestre del año 2019, con un valor real del $11,4 \%$, según cifras oficiales, lo que representa en términos reales a más de 2.524.000 de colombianos sin ninguna posibilidad de vinculación laboral activa (El Tiempo, 2019a).

En este mismo sentido, como consecuencia directa de las escasas oportunidades para acceder a un empleo digno y medianamente remunerado en el sector empresarial, surge la informalidad laboral. Este aspecto viene adquiriendo en Colombia niveles más que alarmantes, dado que cifras estatales reportadas por el Departamento Administrativo Nacional de Estadística (DANE) expresan que, para el año 2018, aproximadamente 10,8 millones de personas, que representan cerca del $48 \%$ de la población económicamente activa en el ámbito urbano y el $86 \%$ en el rural, encontraron en este escenario la única forma de generar ingresos para su sustento y el de sus familias (Dinero, 2016; El Tiempo, 2019b).

La alta informalidad en el país es una de las dificultades más complejas de solucionar, tal y como lo expresa la Organización para la Cooperación y el Desarrollo Económicos (2016). Este fenómeno ha hecho que la productividad nacional sea actualmente en promedio un $25 \%$ menos que hace 50 años, situación que a su vez genera altos niveles de pobreza, especialmente en la población de la tercera edad, que son los que menos oportunidades laborales poseen; y las escasas que ofrece el mercado se caracterizan por ser empleos de baja calidad y con aportes mínimos a los sistemas de seguridad social, pensión e impuestos. Esta situación genera una mayor brecha social, en términos de distribución de ingresos y calidad de vida en la población.

Frente a este aspecto, Quejada, Yánez y Cano (2014) identificaron que la informalidad se concibe desde 3 escenarios: 1) subsistencia (excluidos por productividad menor a la del salario mínimo), 2) voluntaria (excluidos por decisión propia), e 3) inducida (excluidos por regulaciones o segregación laboral), lo que a su vez está influenciado por factores como el género, nivel de formación y edad, como las principales limitantes para que los individuos puedan ingresar a un modelo de contratación formal laboral.

Esta situación afecta y desestimula notoriamente la productividad de todos los sectores de la economía, especialmente el agrícola, el manufacturero y el de construcción, que son los grandes impulsores del empleo en Colombia. Se resalta que el impacto resulta ser mayor en mujeres que en hombres, en un nivel del $60 \%$ frente al $40 \%$, respectivamente. Estos porcentajes son similares con personas afrodescendientes, migrantes o reinsertados de la violencia (Mora, Caicedo \& González, 2017).

Si bien la problemática del desempleo no es nueva en el país, actualmente el panorama más complejo lo viven las personas entre 18 y 28 años de edad, quienes poseen menores oportunidades de vinculación laboral. La cifra supera el millón de colombianos, que representa más de una tercera parte del total de la población desempleada en el entorno nacional (El Tiempo, 2019a).

Con base en los estudios oficiales agenciados por el DANE (2019), para el mes de abril de 2019 la cifra de desempleo juvenil (18 a 28 años) en Colombia alcanzó el $17 \%$, lo que supera notablemente el promedio nacional del $11 \%$. Este panorama afecta en mayor medida al género femenino, con un total de 600.000 mujeres que no cuentan con empleo.

Dentro de este contexto, se ha señalado la escasa vinculación al mercado laboral de las personas jóvenes (18-28 años), por la poca experiencia laboral y la falta de formación técnica o profesional. A estas variables se suma el bajo perfil que poseen, en términos de capacidades frente a las nuevas competencias que demanda el mercado (Ochoa \&Ordóñez, 2004; Sánchez-Castañeda, 2014).

Si bien el acceso a la educación secundaria, técnica o universitaria ha mejorado en el país, esta no cuenta aún con las condiciones para satisfacer el perfil laboral que solicitan las organizaciones, en la medida en que la formación se ha enfocado únicamente en el desarrollo de habilidades duras -conocidas como hard skylls, en inglés-, que hacen énfasis en dotar al individuo de conocimientos y saberes de carácter técnico que 
permiten desarrollar actividades con competencias específicas (Peleckis \& Peleckiene, 2013). Esto se traduce en que no se vincula a jóvenes en Colombia, por no contar con habilidades blandas -o soft skills-, que son competencias asociadas con la capacidad del individuo para, entre otras condiciones, sociabilizar, comunicarse, trabajar en equipo, adaptarse al cambio, solucionar problemas, dominar las TIC, ser tolerantes y asertivos en la toma de decisiones (Cimanti, 2016).

Como lo argumentan Levy y Yadin (2013), las habilidades blandas son entendidas por el mercado laboral como una ventaja competitiva que debe poseer todo el recurso humano, como parte esencial para el desarrollo productivo de cualquier actividad en la organización, en paralelo con las habilidades duras (hard skills), propias de cada profesión o cargo a desempeñar.

En este sentido, la Universidad de Stanford y la Fundación Carnegie Mellon (Sinha, 2008, citado por Mathur, 2017) realizaron un estudio a 500 CEO, para analizar su percepción sobre la importancia de las hard skylls frente a las soft skylls, y establecieron que para el $75 \%$ la condición de éxito laboral se asocia en mayor medida con las habilidades blandas, mientras que solo el $25 \%$ identificó las habilidades duras o técnicas como más importantes dentro de sus empresas.

Consecuente con lo anterior, tanto Human Age Institute (2016) como Neri y Hernández (2019) identifican que los actuales procesos de selección de talento humano incluyen cada vez más la evaluación de habilidades blandas frente a la de habilidades duras, dado que se consideran más difíciles de encontrar. Se estiman también de mayor impacto para las organizaciones, en la medida que facilitan el trabajo en equipo y generan altos niveles de productividad, con rápida adaptabilidad al entorno. A su vez, son más complejas de adquirir, porque involucran aspectos cognitivos, procedimentales y actitudinales.

Por consiguiente, las competencias generales pretenden potencializar factores intrínsecos del individuo que permitan un redescubrimiento de herramientas cognitivas, actitudinales y procedimentales, para responder efectivamente a su entorno, con un sello personal como diferenciador (Frade, 2009, citada por García, 2011). Es preciso potenciar factores que permiten al profesional adaptarse a los cambios respondiendo asertivamente a los retos asumidos.

Es por esto que se debe contar con objetividad y pertinencia en la formación de competencias para enriquecer el perfeccionamiento de los quehaceres laborales mediante el análisis de factores intrínsecos que interactúan entre sí, para que el individuo comprenda la real naturaleza de las situaciones y responda óptimamente a las necesidades que le enmarca el medio (Carrera \& Marín, 2011).

A manera de ejemplo, en los programas de formación asociados con el mundo de la administración de empresas y los negocios, Peleckis y Peleckiene (2013) concluyen que una de las grandes falencias de los recién egresados es no contar con habilidades blandas asociadas con capacidades para desarrollar procesos de negociación, solución de conflictos y emprendimientos. Estos tres elementos resultan esenciales en el perfil laboral que buscan las organizaciones con características actitudinales, inteligencia emocional y liderazgo.

En tal sentido, el modelo de selección y contratación de personal de empresas como eTalent (especialistas en procesos de selección de personal a nivel de Suramérica), tras valorar y filtrar a candidatos con base en los perfiles solicitados por las empresas, eliminan cerca del $70 \%$ de los aspirantes. El motivo: en promedio solo un $30 \%$ se ajusta a los requerimientos básicos de la vacante, de los cuales tan solo un $7 \%$ posee competencias laborales tanto duras como blandas para un desempeño de alto nivel (Evaluar, 2019).

\section{El desafío de formar en competencias laborales generales}

En Colombia, las competencias laborales tradicionalmente estaban asociadas con los programas de formación técnicos ofrecidos por el Servicio Nacional de Aprendizaje (SENA), con una mirada enfocada exclusivamente en el ámbito de formar personas para el trabajo. En este aspecto, Bunk (1994, citado por Guerrero 1999) afirma que se hace una diferenciación del término competencia, a partir del concepto de capacidades profesionales, que se define como un conjunto de elementos que generan destrezas y aptitudes para la correcta realización de actividades propias de una labor o profesión.

Por otra parte, Mertens $(1996,2000)$ menciona que la capacidad para resolver problemas relacionados en el ámbito laboral demanda competencias humanas y sociopolíticas que contribuyan a mejorar la interacción del profesional con el entorno. Estas se clasifican en:

- Básicas: Habilidades que toda persona debe tener para vincularse al campo laboral, tales como lectura, escritura y cálculo.

- Técnico-profesionales o específicas: Relacionadas con la naturaleza propia de la profesión.

- Sociales: Son las que permiten la relación con otros individuos y con el entorno.

- Transversales: Habilidades que complementan planes curriculares, como idiomas e informática, entre otras.

En cuanto a los diseños curriculares que abordan las 
universidades e instituciones de formación laboral, se ha podido establecer que son pocas las que se ocupan de trabajar el tema de capacidades emocionales como un factor necesario para el desarrollo de competencias sociales. En la mayoría de los casos los planes de estudio están enfocados casi siempre en fortalecer exclusivamente los procesos de aprendizaje procedimentales, propios de la labor técnica a desempeñar, y muy poco en los aspectos cognitivos y actitudinales para competencias relacionadas con el manejo de conflictos, comunicación asertiva, motivación, control emocional, emprendimiento, actitudes ciudadanas y sostenibilidad, entre muchos otros (García, 2011).

En Colombia, la propuesta para formar desde la educación básica secundaria en competencias laborales generales surge a partir de la necesidad manifestada por empleadores que afirmaron que los nuevos profesionales egresados de las universidades y centros de formación técnica o tecnológica se caracterizan por tener un buen nivel de formación en el área de laboral de desempeño (hard skills), pero con fuertes deficiencias en las habilidades blandas (soft skills) que resultan necesarias para la competitividad de la empresa, en la medida que a través de ellas se garantizan mayores niveles de estabilidad de los empleados, trabajo en equipo y construcción de un verdadero capital humano, entendido este como un activo financiero cada vez más tangible y valioso para las organizaciones (Vera, 2016).

Para tal fin, el Ministerio de Educación Nacional (2015), tras un importante desarrollo investigativo y numerosas mesas de trabajo, creó un documento que asocia estos elementos mediante lo que se conoce como la "Guía N. 21" (ver Tabla 1), que define el modelo de competencias laborales generales y específicas que se deberían trabajar en las instituciones de educación. Con esta guía, se ha dado respuesta a los cambios laborales organizacionales generados principalmente por el fenómeno de la globalización, mediante un marcado enfoque que se centra inicialmente en crear perfiles que sean más competitivos y, por ende, más atractivos para los procesos de contratación y vinculación laboral, con base en los elementos del ser, saber y hacer.

Para ello, Rojas-Zapata y Hernández-Arteaga (2018) indican que, para desarrollar estas competencias, es necesario que los centros de formación superior capaciten a sus profesores en competencias blandas complementarias, que deben ser independientes a los saberes técnicos de cada profesión, pero complementarias para la vida del estudiante. Solo así, los profesores podrán convertirse en educadores integrales. Tejada (2009) y Rueda (2011), así como Morales y Cabrera (2012) exponen que la docencia y las competencias blandas se adquieren no solo dentro del aula de clase, sino también en el contexto natural de la profesión, por lo que el ejercicio docente debe crear mayor número de espacios en los entornos empresariales reales que le esperan al futuro egresado.

Lo anterior comprueba entonces que lograr gestionar de manera adecuada este tipo de competencias en los ámbitos académicos permitirá contribuir a un mejor futuro profesional del egresado, dado que contará con mayores capacidades de liderazgo, resolución de conflictos, comunicación asertiva, negociación y adaptación al contexto laboral. Estos aspectos resultan altamente relevantes si se trata de encontrar la primera oportunidad de empleo en un país en el que son escasos los puestos de trabajo.

Tabla 1

Competencias laborales generales (MEN)

\begin{tabular}{ll}
\multicolumn{1}{c}{ Competencias } & \multicolumn{1}{c}{ Habilidades } \\
\hline 1. Personales & $\begin{array}{l}\text { Orientación ética, dominio personal, inteligencia emocional y adaptación al cambio. } \\
\text { Toma de decisiones, creatividad, solución de problemas, atención, memoria y concentración. } \\
\text { 3. Intelectuales }\end{array}$ \\
4. Intrapersonales & $\begin{array}{l}\text { Identifica oportunidades de crear empresa, unidades de negocios, consecución de recursos, } \\
\text { análisis del riesgo, gestión en mercadeo. } \\
\text { Comunicación, trabajo en equipo, liderazgo, manejo de conflictos, capacidad de adaptación, } \\
\text { proactividad. }\end{array}$ \\
5. Organizacional & $\begin{array}{l}\text { Gestión de información, orientación al servicio, referenciación competitiva, gestión de } \\
\text { recursos y responsabilidad ambiental. } \\
\text { Identifica, transforma, innova, uso de herramientas informáticas y tecnológicas, creatividad, } \\
\text { apropiación de conocimiento. }\end{array}$ \\
\hline
\end{tabular}

Fuente: Tomado del Ministerio de Educación Nacional (2015).

\section{Discusión}

Se observa como resultado de esta revisión que las universidades deben enfocar mayores esfuerzos hacia un diseño curricular más robusto en el desarrollo de habilidades blandas, particularmente las enfocadas a identificar oportunidades de negocio y creación de empresas, como una alternativa diferente de opción laboral. Elementos como la capacidad de trabajo en equipo, asertividad, manejo de conflictos, capacidad de 
adaptación, habilidades comunicativas, dominio de las TIC, tecnología, innovación, creatividad e investigación son ejes de trabajo que no pueden ser tratados como simples electivas complementarias al currículo disciplinar, sino como un tema fundamental para el nuevo perfil profesional del siglo XXI.

En definitiva, con base en el trabajo de Human Age Institute (2016), se identificaron las competencias generales que deberían estar implícitas en cualquier currículo académico de instituciones de educación superior, que se pueden observar en la Figura 1. Allí se contextualizan las competencias de mayor demanda por parte de las organizaciones en un entorno global. También se puede observar que las habilidades sociales son las más solicitadas particularmente en los temas de resolución de problemas, orientación a objetivos, colaboración, toma de decisiones y aprendizaje activo, como elementos claves que debe poseer un profesional para desempeñarse de manera eficaz.

Igualmente, se identificó que, de acuerdo con cada generación, el desarrollo de competencias laborales generales es diferente y se potencializa en mayor o menor medida con base en sus expectativas de vida laboral. En gran medida estas competencias son comunes a todas las generaciones, entre las cuales destacan la orientación al servicio y la solución de problemas como las más representativas.

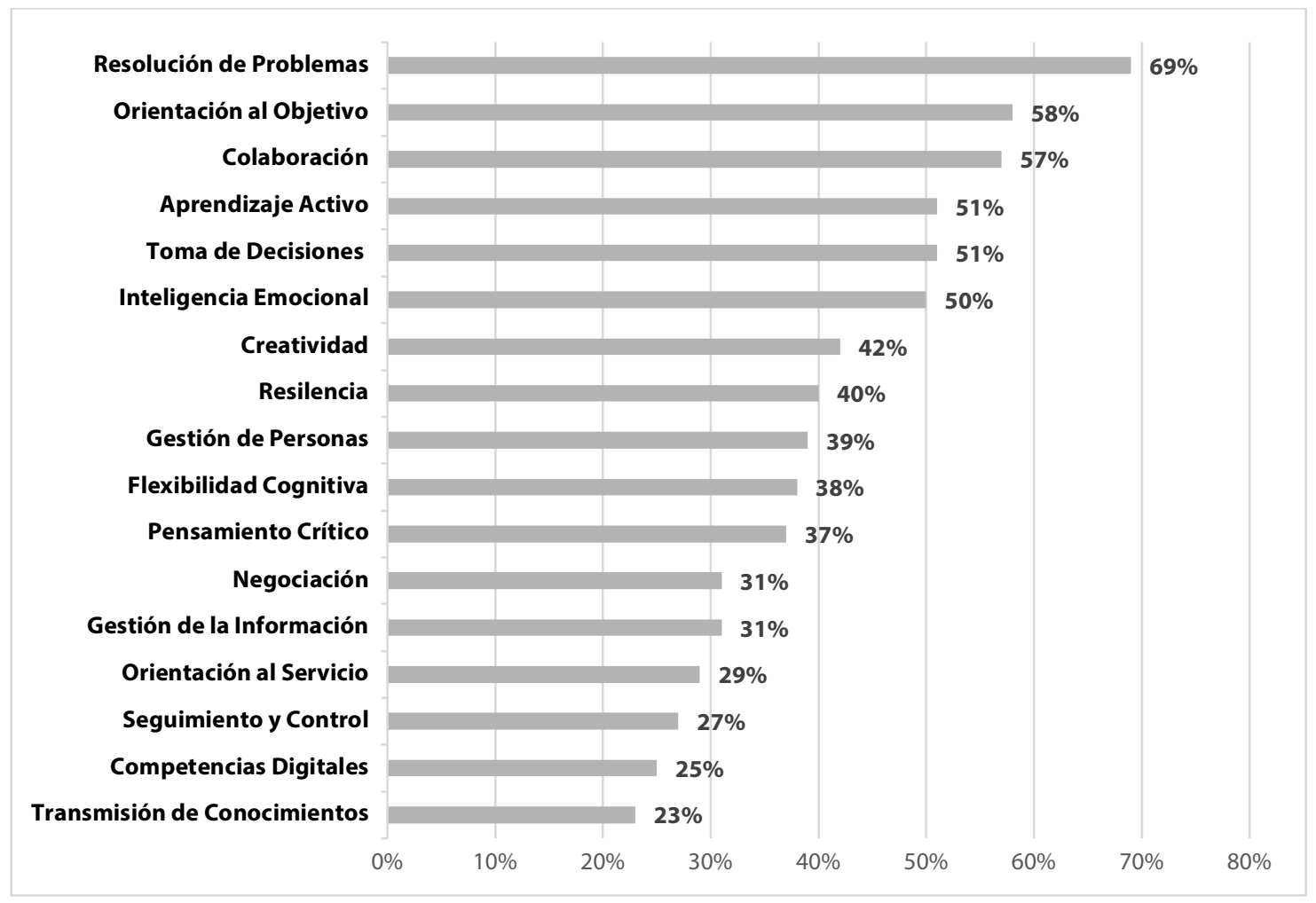

Figura 1. Habilidades blandas solicitadas por las empresas. Fuente: Estudio Soft Skills 4 Talent (Human Age Institute, 2016).

No obstante, para el caso de la llamada generación $X$ (años 1965-1980), su mayor potencial está en el desarrollo de las habilidades asociadas con negociación, gestión de personas, toma de decisiones e inteligencia emocional, mientras que para los llamados millennials (1981-2004) el aprendizaje activo, gestión de información, creatividad y resiliencia prevalecen como sus mayores fortalezas. Por su parte, para la generación de los baby boomers (años 1946-1964) las capacidades que mayores niveles alcanzan son la de gestión de personas y la de negociación. Los resultados también demuestran que la competencia de conocimientos digitales debe ser desarrollada por todo tipo de generación.

\section{Conclusiones}

Teniendo en cuenta el modelo formativo que actualmente desarrolla la mayoría de los centros de educación superior en Colombia, es necesario analizar las debilidades que limitan la evolución de la enseñanza basada en el tradicional enfoque técnico del conocimiento hacia uno más completo, que incluya un verdadero enfoque formativo basado en competencias, tanto duras como blandas, lo que permitiría obtener un modelo de enseñanza y aprendizaje capaz de ofrecer al mercado laboral un perfil profesional con mayores capacidades para el pensamiento crítico, percepción y manejo de 
las emociones. Un perfil que le permita al individuo observar, comprender, experimentar, aprender y proponer soluciones a los desafíos que impone el sector real empresarial.

El crear nuevas capacidades y competencias en los egresados es de vital importancia para lograr un perfil profesional adecuado para el entorno empresarial colombiano, especialmente en factores que potencialicen habilidades sociales. Así que, es momento oportuno para fortalecer los proyectos educativos institucionales con nuevos modelos de enseñanza y aprendizaje, que resulten más propicios para impulsar capacidades de emprendimiento como alternativa de trabajo y opción de vida para los egresados.

Con base en el fenómeno de disminución de nuevos estudiantes que presentan varias profesiones, resulta oportuno que la universidad colombiana reflexione en forma crítica sobre los contenidos temáticos que se imparten en sus aulas y la aplicabilidad de estos. En muchos casos ciertas asignaturas pierden validez frente al contexto actual de la globalización y el perfil laboral que buscan las empresas, para reenfocar esfuerzos en el desarrollo de nuevos espacios académicos que reformulen las competencias duras y blandas con las que pueda contar el egresado para ser competitivo en el mercado laboral.

Por tanto, resulta oportuno y necesario generar un mayor número de investigaciones que den soporte a un rediseño curricular innovador en cada uno de los diferentes programas de educación superior. Para este fin se hace necesaria una interacción dinámica y permanente entre empresa y universidad, a fin de identificar en mejor medida las necesidades que el sector real demanda en términos de competencias laborales.

\section{Referencias}

Águila, E., Mejía, N., Pérez, F., Rivera, A., \& Ramírez, E. (2015). Pobreza y vulnerabilidad en México: caso de jóvenes que no estudian ni trabajan. Revista Estudios Económicos, 30(1), 3-49.

Banco de la República. (2019). Boletín de Indicadores Económicos junio de 2019. Recuperado de http:// www.banrep.gov.co/economia/pli/bie.pdf

Bunk, O. P. (1994). La transmisión de las competencias en la formación y peífeccionamiento profesionales de la RFA. Formación Profesional. Revista Europea, 1/1994, 8-14.

Carrera, C., \& Marín, R. (2011). Modelo pedagógico para el desarrollo de competencias en educación superior. Revista Electrónica Actualidades Investigativas En Educación, 11(1), 1-32.

Cimanti, B. (2016). Definition, development, assessment of soft skills and their role for the quality of organizations and enterprises. International Journal Quality Research, 10(1), 97-130.

Departamento Administrativo Nacional de Estadística. (2019). Gran encuesta integrada hogares (GEIH) Mercado laboral. Recuperado de http://bit.ly/2KKFz9b

Departamento Administrativo para la Prosperidad Social. (2019). Componente habilidades para la vida, programa jóvenes en acción - JEA. Bogotá, Colombia.

Dinero. (2016). Las devastadoras consecuencias de la informalidad en Colombia. Dinero. Recuperado de http://bit.ly/2lftFTI

El Tiempo. (2019a). Informalidad de trabajadores no cede y es de $48,2 \%$ según DANE. Recuperado de http://bit. ly/2R7lqdu

El Tiempo. (2019b). La informalidad laboral del país es uno de los problemas más graves que enfronta el país. 06 abril de 2019. Recuperado de. El Tiempo. Recuperado de http://bit.ly/2WDzl26

Evaluar. (2019). Tu proceso de reclutamiento y selección simple, inteligente y moderno. Recuperado de https:// www.evaluar.com/

Frade, L. (2009). Desarrollo de competencias en educación: desde preescolar hasta el bachillerato. México, DF: Inteligencia Educativa.

García, J. (2011). Modelo educativo basado en competencias: importancia y necesidad. Revista Electrónica Actualidades Investigativas En Educación, 11(3), 1-24.

Guerrero, A. (1999). El enfoque de las competencias profesionales: una solución conflictiva a la relación entre formación y empleo. Revista Complutense de Educación, 10(1), 335-360.

Guirao, G. (2015). Utilidad y tipos de revisión de literatura. Revista de Enfermería, 9(2), 64-75.

Human Age Institute. (2016). Soft skills 4 talent 2016. London, U.K; Manpower.

Levy, I., \& Yadin, A. (2013). Soft Skills: An Important Key of Employability. International Journal of E-Education, e-Business, e-Management and e-Learning, 3(5), 145-156.

Mathur, A. (2017). The Role of Soft Skills in Enhancing Employability of Technical Graduates. A Study. International Journal on Emerging Technologies, 8(1), 65-67.

Mertens, L. (1996). Competencia laboral: sistemas, surgimiento y modelos. Montevideo, Uruguay; Cinterfor/OIT.

Mertens, L. (2000). La Gestión por Competencia Laboral en la Empresa y la Formación Profesional. Madrid, España: ediciones OEI.

Ministerio de Educación Nacional. (2005). Articulación de la educación con el mundo productivo. Competencias laborales generales. Guia \#21. Bogotá, Colombia: Imprenta Nacional de Colombia.

Mora, J., Caicedo, C., \& González, C. (2017). The Duration of Youth Unemployment and the "Ninis" in Cali. Revista de Economía Institucional, 19(33), 167-184.

Morales, R., \& Cabrera, J. (2012). Competencias docentes transversales, el método de selección. REDU Revista 
de Docencia Universitaria, 10(2), 75-101.

Neri, J. C., \& Hernández, C. A. (2019). Los jóvenes universitarios de ingeniería y su percepción sobre las competencias blandas. Revista Iberoamericana Para La Investigación y EI Desarrollo Educativo, 9(18), 768-791.

Ochoa, D., \& Ordóñez, A. (2004). Informalidad en Colombia. Causas, efectos y características de economía del rebusque. Revista Estudios Gerenciales, Universidad ICESI, 20(90), 105-118.

Organización para la Cooperación y el Desarrollo Económicos. (2016). Estudios económicos de la OCDE Colombia. Resumen ejecutivo para Colombia. Publicaciones OCDE.

Peleckis, K., \& Peleckiene, V. (2013). Developing negotiation competencies of business management students: structural and content dimensions. Review Business: Theory and Practice, 14(4), 346-357.

Quejada, R., Yánez, M., \& Cano, K. (2014). Determinantes de la informalidad laboral, caso Colombia. Revista Investigación \& Desarrollo, 22(1), 126-145.

Rojas-Zapata, A. F., \& Hernández-Arteaga, I. (2018). Competencias docentes: en los lineamientos institucionales y de programa. I+ $D$ Revista de Investigaciones, 12(2), 27-36. https://doi. org/10.33304/revinv.v12n2-2018003

Rueda, J. F. (2011). La Profesionalización, Elemento Clave del Éxito de la Empresa Familiar. Revista Visión de Futuro, 15(1), 67-84.

Sánchez-Castañeda, A. (2014). Los jóvenes frente al empleo y el desempleo: la necesaria construcción de soluciones multidimensionales y multifactoriales. Revista Latinoamericana de Derecho Social, 18(4), 133-162.

Tejada, F. (2009). Competencias docentes del Profesorado. Revista de Currículum y Formación Del Profesorado., 13(2), 1-15.

Vera, F. (2016). Infusión de habilidades blandas en el currículo de la educación superior: clave para el desarrollo de capital humano avanzado. Revista Akademeia, 7(1), 53-73. 